\section{The Amsler chart is of doubtful value in retinal screening for early laser therapy of subretinal membranes. The West London Survey}

FH Zaidi, R Cheong-Leen, EJ Gair, R Weir, E Sharkawi, N Lee and K Gregory-Evans

\section{Abstract}

Purpose Validating the current protocol of Amsler chart grid surveillance for the early detection of subretinal neovascular membrane (SRN) in age-related macular degeneration (AMD), and investigating its value in facilitating early laser therapy.

Methods A retrospective pilot study. Setting: central London eye hospital with dedicated 24-h ophthalmic casualty serving West and West-central London. Participants: 100 consecutive AMD patients who attended casualty with vision loss fulfilling the following criteria: patients had received and been instructed in the use of Amsler charts according to the unit's dispensation protocol, fluorescein angiography which confirmed new SRN. Patients presented over 20 months. Outcome measures were detection of SRN by the Amsler chart, and laser treatment of SRN. Results The Amsler chart surveillance protocol had detected SRN in 29 of the 100 patients. The surveillance protocol detected less than $30 \%$ of the specific patients who subsequently underwent laser treatment. A statistically significant difference was seen on comparing the ages of patients in whom the screening protocol was successful versus those in whom it was unsuccessful (student's $t$-test, $\left.P<3.2 \times 10^{-13}\right)$. Younger patients were more likely to be detected using the Amsler chart. A one-tailed $\chi^{2}$ test approached, but did not achieve, statistical significance $\left(\chi^{2}=1.057\right.$, $P<0.3)$ suggesting that patients who have already lost vision to SRN in one eye might not be more likely to be detected using the surveillance protocol than patients in whom SRN was affecting their first eye. In all, $38 \%$ of surveillance responders went on to receive laser therapy, compared with $37 \%$ of surveillance nonresponders.

Conclusions The current Amsler chart surveillance protocol is suboptimal for detecting SRN in AMD, and a proportion of cases suitable for early laser therapy may be missing rapid detection. The results are especially important since recent advances in laser therapy for SRN require early detection for optimal effectiveness.

Eye (2004) 18, 503-508. doi:10.1038/sj.eye.6700708

Keywords: Amsler chart; Amsler grid; photodynamic laser therapy; argon laser; transpupillary thermotherapy; pupillometry; imaging; psychophysical tests; screening; agerelated macular degeneration

\section{Introduction}

Many severe cases of age-related macular degeneration (AMD) are due to the development of sub-retinal neovascular membrane (SRN). ${ }^{1-8}$ SRN has also received attention because it is potentially treatable, with Argon laser ${ }^{8,9}$ and recently transpupillary thermotherapy (TTT) ${ }^{10-13}$ or photodynamic therapy with verteporfin (PDT) ${ }^{6,7}$ Early detection of SRN is required to increase the effectiveness of these treatments. ${ }^{6-8,13}$

For the last six decades, to detect SRN early, most ophthalmologists have asked AMD patients to undergo a surveillance protocol using a printed diagram called an Amsler chart (also called Amsler grid). ${ }^{14,15}$ After being given instructions on regular use of the Amsler chart, patients self-screen their own vision at home. If positive, the formerly straight lines on the chart
The Western Eye Hospital Marylebone Road London, UK

Correspondence: FH Zaidi Department of Ophthalmology Faculty of Medicine Imperial College London Laboratory Block Charing Cross Campus Fulham Palace Road London W6 8RF, UK Tel: + 442083833696 Fax: +44208383 3651 E-mail: fhz12@

hotmail.com

Received: 13 November 2002 Accepted: 21 March 2003

This study was presented at the Royal College of Ophthalmologists Annual Congress, The International Convention Centre (ICC), Birmingham, England, May 2003, under the title 'Effectiveness of detecting subretinal membranes in age-related macular degeneration';

psychophysical data arising from this study was included in a presentation at the Imperial Neuroscience and Psychological Medicine Annual Research Meeting, London, England, May 2003, under the title 'Screening of vision in AMD.' 
either seem distorted or absent, and this heralds the development of SRN. There are three key components to this form of surveillance. Firstly, charts are given to all patients with AMD together with good instructions on its use, particularly to return urgently to the eye department if distortion or field loss develops. Secondly, patients use the chart at least once a week. Thirdly, patients actually act in accordance with instructions.

There have been a few medium-sized studies to validate Amsler charts as a surveillance tool in retinal disease, ${ }^{15-19}$ but comparatively little data exists on the benefits of the Amsler chart protocol in patients with AMD who go on to develop SRN, specifically whether the protocol facilitates detection to the degree of improving treatment outcome. With new treatment strategies so dependent on early detection, we elected to assess the effectiveness of this standard surveillance protocol, especially with this context in mind.

\section{Methods}

The Amsler chart surveillance protocol that we studied is typical of that used in eye departments in the British National Health Service. We undertook a retrospective, pilot study to validate the effectiveness of the Amsler chart for early detection of SRN in AMD, and subsequent initiation of laser treatment. The objective was, firstly, to estimate what proportion of AMD patients who have been given the Amsler chart have subsequent SRN detected because of using the chart. Secondly, we looked at how Amsler chart use affected treatment outcome.

Recruitment was undertaken retrospectively from patients who had attended the dedicated 24-h ophthalmic casualty department at the Western Eye Hospital serving West and West-Central London, which includes referrals from St Mary's, Hammersmith, Charing Cross, Hillingdon, Chelsea and Westminster Hospitals.

Data was collected from 100 consecutive AMD patients, who had attended over a 20 month period, and who fulfilled two inclusion criteria: they had all been given and instructed in the use of the Amsler chart according to unit protocol, and had subsequently developed SRN. The following information was also collected: age; presenting complaint (eg the patient had noticed Amsler chart abnormalities); mode of presentation (self-referral, from a routine clinic appointment, via optometrist or general practitioner (GP); whether this was the patient's first or second eye to be affected by SRN; and the outcome of attendance: was laser treatment initiated and what type had been used. SRN diagnosis was established by subsequent fundus fluorescein angiography (FFA). Angiograms were interpreted by one of two consultant ophthalmologists with special retinal expertise (KGE \& NL). All 100 study patients had received a standard Amsler chart (chart number one by Keeler ${ }^{\mathbb{R}}$ ) at this unit, with instructions on how to use it and what to do (attend the eye department) if they noticed changes. Each small square when held at $1 / 3 \mathrm{~m}$ subtends 1 degree of arc. Specific instructions were given on how to use the chart at the time of its dispensation: 'Hold the chart before yourself at 30 centimetres, wearing spectacles as required, and test each eye separately. Use the chart at least once a week. Focus on the spot in the centre and observe the lines'. Patients were instructed to urgently return to the hospital's eye casualty (accident and emergency (A/E)) if they noticed any new bending or gap in the lines.

Those patients who had attended casualty because they had noticed Amsler chart changes were termed 'responders'. Those who had attended but not because of observations made using their Amsler charts were termed 'nonresponders'.

\section{Results}

Key results are summarised in Table 1 and Figure 1. Of 100 AMD patients who had been recruited into the surveillance protocol and had subsequently developed SRN, attendance at casualty had been precipitated by Amsler chart use in only 29 patients.

\section{Effect of age}

The mean age of responders was approximately 61 and nonresponders approximately 77 (Table 1). A greater proportion of older patients failed to respond to the Amsler chart: student $\mathrm{t}$-test comparing the ages of Amsler responders and nonresponders was statistically significant $\left(P<3.2 \times 10^{-13}\right)$.

\section{Presenting with symptoms in the first vs the second eye}

The total number of patients having already lost vision to SRN in one eye and who now developed it in a second eye was 21 . Most of these $(13,62 \%)$ had not attended because of Amsler chart changes. However, when comparing the number of patients presenting with second eye symptoms between the responder and nonresponder subgroups, a one-tailed $\chi^{2}$ test did not quite achieve statistical significance $\left(\chi^{2}=1.057, P<0.3\right)$.

\section{Mode of presentation}

Out of 29 Amsler responders, only 18 had actually followed the protocol and presented direct to the eye casualty. The others had first presented to their optometrist or GP (Table 1 and Figure 1). Interestingly, of 
Table 1 AMD patients who develop SRN while undergoing an Amsler chart surveillance protocol, comparing those who attended the eye casualty because they had noticed chart changes (responders) with those who attended for other reasons (nonresponders)

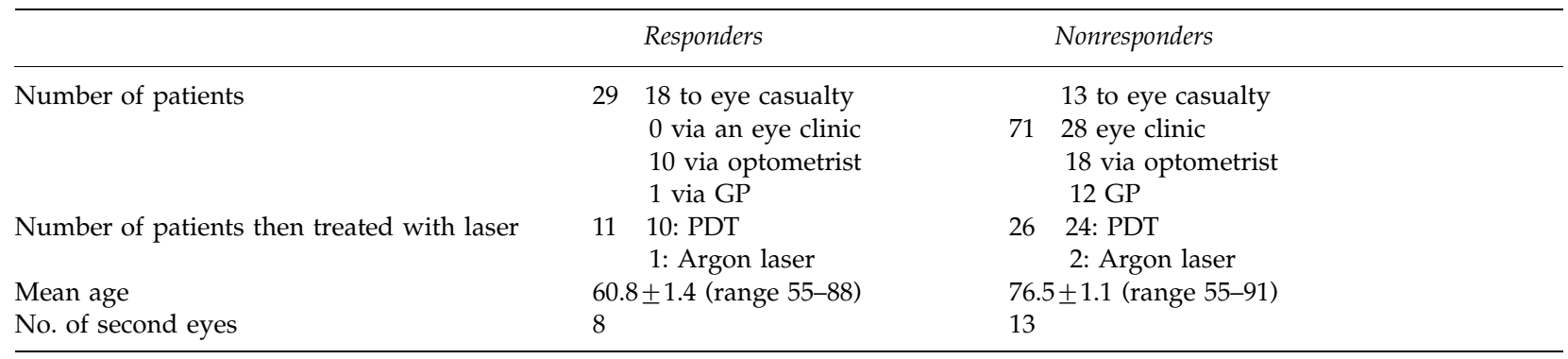

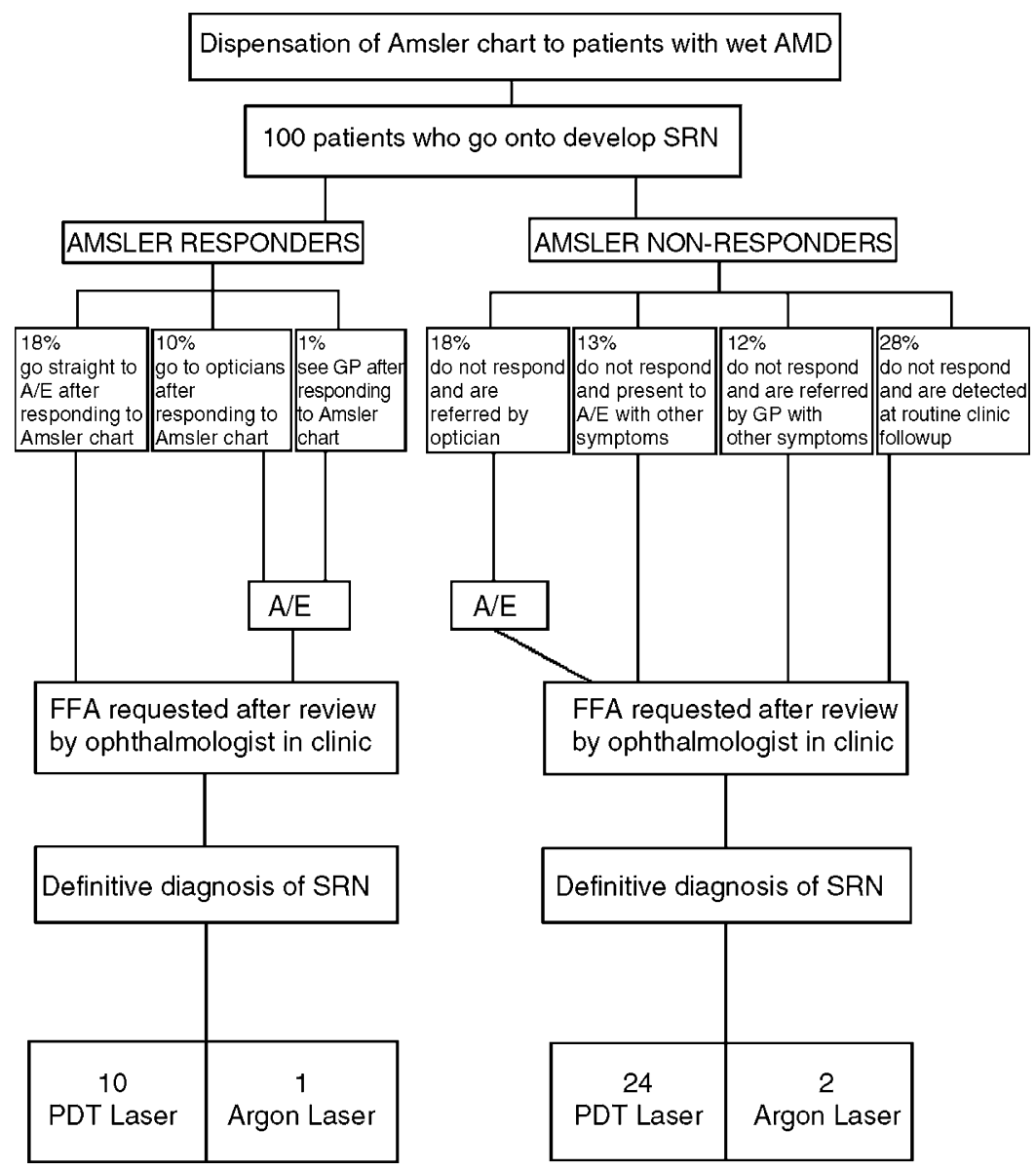

Figure 1 Flow diagram illustrating mode of presentation and outcomes for study patients.

the 71 nonresponders 13 (18\% of nonresponders) did present direct to casualty but not because of Amsler chart use (Table 1 and Figure 1). The largest subgroup of nonresponders $(28,39 \%$ of nonresponders) had in fact been referred for urgent management from routine ophthalmic clinic follow-up (Table 1 and Figure 1).

\section{Therapeutic outcome}

Of the 100 patients, 37 went on to receive laser treatment. Less than a third of these treatments (11 of 37) had been precipitated by Amsler chart surveillance. This proportion of patients in whom surveillance with the 


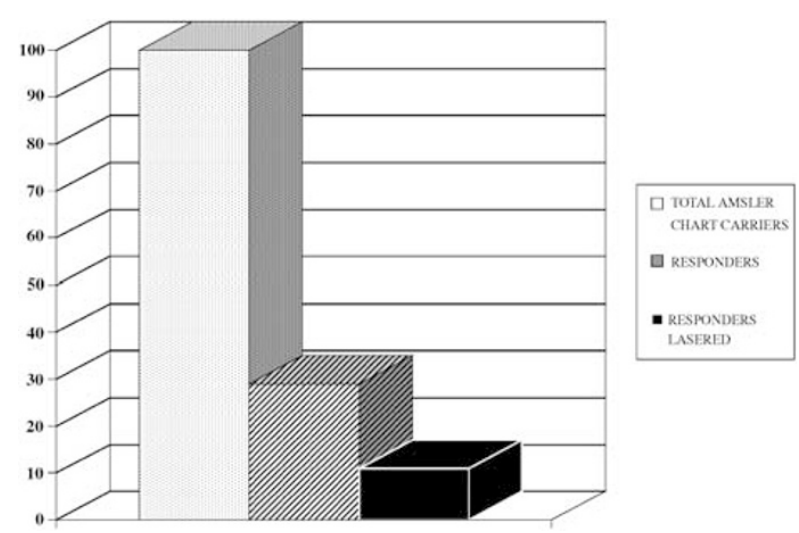

Figure 2 Breakdown of positive outcomes in which the Amsler chart led to the initiation of laser treatment.

Amsler chart made a difference to outcome, are illustrated in Figure 2. Treated patients included 38\% of responders and $37 \%$ of nonresponders. Most had undergone photodynamic therapy with verteporfin (Table 1 and Figure 1).

\section{Discussion}

This study has found that in a population of patients with AMD complicated by SRN, Amsler chart surveillance led to appropriate casualty presentation in less than one in three and laser treatment in only $11 \%$ (Figure 2). The majority of patients presented for other reasons despite being incorporated into the surveillance protocol.

Of the parameters studied age was found to have the most statistically significant effect on outcome, with Amsler chart surveillance more effective in younger patients. Interestingly, we did not find that people in whom SRN was affecting their second eye were more likely to be detected using the Amsler chart. This counter-intuitive result may however be a consequence of the study size. Many aspects of the Amsler chart surveillance protocol were not studied, including the number of AMD patients who are enrolled into the protocol yet do not develop SRN, those who remain undetected, the number and outcomes of casualty attendances precipitated by the protocol that do not result in a diagnosis of SRN, and a breakdown of symptomatology in nonresponders to the protocol. These will be included in future studies precipitated by this pilot project. Another adverse factor in this study may be that it was retrospective. Since, however, a major factor in the study is compliance, ${ }^{16}$ this might have been artificially enhanced by a prospective study.
To varying degrees other studies have questioned the validity of Amsler chart screening. ${ }^{15,19}$ None of the other larger studies looked exclusively at SRN in AMD, but included patients with other conditions such as high myopia and ocular histoplasmosis. ${ }^{16}$ Also, treatment outcome was not investigated and some investigators used different protocols to the current study, for example, patients had used an illuminated Amsler chart. ${ }^{17,18}$ These previous studies did however suggest two principle problems with Amsler chart screening: difficulty with compliance, ${ }^{16}$ and problems with the subjective nature of the test. ${ }^{19}$ Either of these problems may explain the age-dependent success rate seen in this study. Relatively high levels of concentration are needed to undertake the test and levels of fatigue and anxiety are important, compounded by the perceptual completion phenomenon wherein results have been shown to vary during the course of the day. ${ }^{19}$ All three are often affected by age, to varying degrees depending on the individual. Anxiety in the subject can also influence the results of testing.

The current study is also unique as we have looked at treatment outcome. This was however limited to documenting whether patients underwent laser treatment. Other therapeutic outcomes, such as benefits from prescribing low vision aid, blind registration, etc were not investigated. Also, because this is only an initial pilot study, visual benefit from laser treatment was not documented. Despite this it is particularly notable that a similar proportion of surveillance responders and nonresponders were suitable for laser treatment (respectively $38 \%$ compared with $37 \%$ ). This might suggest that Amsler chart surveillance is not only of limited value in precipitating casualty attendance when SRN develops, but is also poor at identifying SRN that is amenable to treatment. This although is not surprising since it has been shown that discrimination between 'treatable' and 'untreatable' $\mathrm{SRN}$ is difficult even when fluorescein angiography has been undertaken. ${ }^{20}$

These results are of increasing importance however since, in Britain, the National Institute for Clinical Excellence has highlighted early diagnosis as vital to the outcome of photodynamic therapy in SRN. ${ }^{6}$ Argon laser is also most effective when SRN is detected early. ${ }^{9}$ Further subgroups who stand to benefit from the chart are being investigated on the basis of new questions raised by this study, including the relationship between detection and the subtype of subretinal membrane in terms of morphology, size, and location. This might itself be closely linked to visual function at the time the chart was issued, at the time of detection, and following laser therapy. 
To some extent, the problems of undertaking an effective screening protocol for SRN in AMD are surmountable. Compliance can be increased through intensifying surveillance. This could involve compiling an 'at risk' register of those with AMD and sending these patients reminders on using the Amsler chart at regular intervals. Giving written instructions on the use of the chart could also increase compliance. However, the problem of the test's subjective nature would still remain. Alternatives to the standard paper Amsler chart, as used in the British National Health Service, might also make the screening protocol more objective. These would require more frequent clinical assessments but, with appropriate equipment and training, some could be undertaken in a primary care setting such as in GP or optometrist clinics. This could involve regular assessments with an objective screening tool such as retinal photography ${ }^{21}$ or optical coherence tomography. For use in the present the former is the most promising, and is used already in diabetes ${ }^{21}$ and glaucoma clinics. However, a major drawback is that these imaging tools do not function in the presence of significant media opacities, which are common in the elderly. Other tools such as visual perimetry, illuminated and suprathreshold Amsler grids, and colour-contrast sensitivity are subjective, and thus likely to suffer from this same weaknesses found with the standard Amsler chart, while red foveal flicker fusion frequency has rarely been tried outside research. ${ }^{17,18,22,23}$ Pupillometry ${ }^{24}$ offers promise for the future as it is eminently objective and still functions in the presence of almost all significant media opacities, but the potentially complex and very specialised technology is not suitable for operation by GPs, optometrists, and ophthalmologists untrained in its use, and the advanced pupillometry which could be used in retinal diagnosis demands more technical skill and expertise in data interpretation than is possessed by even most ophthalmologists familiar with its general use. However, any of these potential community screening protocols would benefit greatly from telemedicine links which could feed into regional centres and thus allow supervision 'from a distance' by ophthalmologists experienced in these retinal and/or technical fields, who would also interpret the results of the more complex and overtly specialised imaging or physiological recording techniques. Alternately, a regional centre could provide a centralised service for patients at 6-month intervals, out of which also operated mobile surveillance units for house-bound or noncompliant patients, which could be aided by telemedicine.

Nevertheless, in the absence of a validated alternative being routinely implemented, the Amsler chart continues to offer inexpensive rudimentary serveillance with a pick-up rate of almost one in three patients with SRN in this study, and should thus continue to be dispensed.
Bearing in mind the prevalence of AMD and the increased therapeutic importance of early detection of SRN, it is clear that improvements in the current surveillance protocol are required. Moving screening into primary care with new technology, supported by telemedicine links to dedicated specialists offering technical expertise in hospitals, might provide an opportunity to construct a more effective, coordinated, national screening protocol. A national screening programme of this nature would be a major development in clinical ophthalmology and a logical outcome if the field of laser/surgical therapy for AMD continues to advance.

\section{Acknowledgements}

We would like to thank Mr Karl Southerton, The Western Eye Hospital, for his extensive technical contribution. The authors have no proprietary interest in any aspect of this study.

\section{References}

1 Leibowitz HM, Krueger DE, Maunder LR, Milton RC, Kini MM, Kahn HA et al. The Framingham Eye Study monograph: An ophthalmological and epidemiological study of cataract, glaucoma, diabetic retinopathy, macular degeneration, and visual acuity in a general population of 2631 adults, 1973-1975. Surv Ophthalmol 1980; 24(Suppl): 335-610.

2 Hall NF, Gale CR. Prevention of age related macular degeneration. BMJ 2002; 325: 1-2.

3 Thompson JR, Du L, Rosenthal AR. Recent trends in the registration of blindness and partial sight in Leicestershire. Br J Ophthalmol 1989; 73: 95-99.

4 Bruce I, McKennell A, Walker E. Blind and Partially Sighted Adults in Britain: The RNIB Survey, Vol. 1. HMSO: London, 1991, pp 43-44.

5 Evans J. Causes of blindness and partial sight in England and Wales 1990-1991. Office of Population Censuses and Surveys, Studies on Medical and Population Subjects, Number 57 HMSO: London, 1995.

6 National Institute for Clinical Excellence. Photodynamic Therapy for Age-related Macular Degeneration, Consultation Document, 14 October 2002.

7 Bressler NM. Age related macular degeneration. New hope for a common problem comes from photodynamic therapy. BMJ 2000; 321: 1425-1427.

8 Fine SL. Early detection of extrafoveal neovascular membranes by daily central field evaluation. Ophthalmology 1985; 92: 603-609.

9 Walsh AW, Magargal LE, Wright F, Donoso LA. The early natural history of subfoveal neovascular membranes in eyes with age-related macular degeneration. Ann Ophthalmol 1989; 21: 348-350.

10 Newsom RS, McAlister JC, Saeed M, McHugh JD. Transpupillary thermotherapy (TTT) for the treatment of choroidal neovascularisation. Br J Ophthalmol 2001; 85: 173-178. 
11 Rogers AH, Reichel E. Transpupillary thermotherapy of subfoveal occult choroidal neovascularization. Curr Opin Ophthalmol 2001; 12: 212-215.

12 Reichel E, Berrocal AM, Ip M, Kroll AJ, Desai V, Duker JS et al. Transpupillary thermotherapy of occult subfoveal choroidal neovascularization in patients with age-related macular degeneration. Ophthalmology. 1999; 106: 1908-1914.

13 Algvere PV, Seregard S. Age-related maculopathy: pathogenetic features and new treatment modalities. Acta Ophthalmol Scand 2002; 80: 136-143.

14 Marmor MF. A brief history of macular grids: from Thomas Reid to Edvard Munch and Marc Amsler. Surv Ophthalmol 2000; 44: 343-353.

15 Schuchard RA. Validity and interpretation of Amsler grid reports. Arch Ophthalmol 1993; 111: 776-780.

16 Fine AM, Elman MJ, Ebert JE, Prestia PA, Starr JS, Fine SL. Earliest symptoms caused by neovascular membranes in the macula. Arch Ophthalmol 1986; 104: 513-514.

17 Miller D, Lamberts DW, Perry HD. An illuminated grid for macular testing. Arch Ophthalmol 1978; 96: 901-902.

18 Achiron LR, Witkin NS, McCarey B, Primo S. The illuminated high contrast macular grid: a pilot study. J Am Optom Assoc 1995; 66: 693-697.
19 Achard OA, Safran AB, Duret FC, Ragama E. Role of the completion phenomenon in the evaluation of Amsler grid results. Am J Ophthalmol 1995; 120: 322-329.

20 Holz FG, Jorzik J, Schutt F, Flach U, Unnebrink K. Agreement among ophthalmologists in evaluating fluorescein angiograms in patients with neovascular age-related macular degeneration for photodynamic therapy eligibility (FLAP-study). Ophthalmology 2003; 110: 400-405.

21 Eiser JR, Eiser C, Riazi A, Taylor DJ, Hammersley S, Tooke JE. Screening for diabetic retinopathy is well received by patients and may improve self-management intentions. Diabet Med 2001; 18: 835-841.

22 Richer SP. Is there a prevention and treatment strategy for macular degeneration? J Am Optom Assoc 1993; 64: 838-850.

23 Wall M, Sadun AA. Threshold Amsler grid testing. Crosspolarizing lenses enhance yield. Arch Ophthalmol 1986; 104: 520-523.

24 Volpe NJ, Plotkin ES, Maguire MG, Hariprasad R, Galetta SL. Portable pupillography of the swinging flashlight test to detect afferent pupillary defects. Ophthalmology 2000; 107: 1913-1921. 